\title{
Enabling Storytelling by Aphasics in an Augmented Home Environment
}

\author{
Abdullah Al Mahmud \\ Dept. Of Industrial Design \\ Eindhoven University of Technology \\ Den Dolech 2, 5600 MB, Eindhoven \\ The Netherlands \\ $+31402472147$ \\ a.al-mahmud@tue.nl
}

\author{
Dzmitry Aliakseyeu \\ User Experiences Group \\ Philips Research Europe \\ HTC 34, 5656AE, Eindhoven \\ The Netherlands \\ +31402747775 \\ d.aliakseyeu@gmail.com
}

\author{
Jean-Bernard Martens \\ Dept. Of Industrial Design \\ Eindhoven University of Technology \\ Den Dolech 2, 5600 MB, Eindhoven \\ The Netherlands \\ $+31402475208$ \\ j.b.o.s.martens@tue.nl
}

\begin{abstract}
We present the design of a system for Aphasics that assist them in storytelling. Storytelling, in the sense of being able to relate recent and past experiences to relevant others, is considered to be crucial for the quality of life and psychological wellbeing of most people. The storytelling support system that we propose is primarily intended to be used by Aphasics in their postrehabilitation period. Our focus is on the creation of daily stories with the help of passively captured materials, hence emphasizing the need for fairly effortless interaction from the side of the end user. End-user requirements gathering was especially difficult in this project, because of the verbal limitations in our user group. Many requirements for the system were hence decided through consulting proxies of the Aphasics, such as their caregivers. The preliminary feedbacks on our design provide interesting insights for the design of a more complete storytelling system for Aphasics. We also discuss some interesting challenges from a technological and methodological point of view that arose in the course of the design.
\end{abstract}

\section{Categories and Subject Descriptors}

K.4.2 [Computers and Society]: Social Issues- Assistive technology for persons with disabilities

\section{General Terms}

Design, Experimentation, Human Factor.

\section{Keywords}

Aphasia, Augmentative and Alternative Communication (AAC) devices, Sharing experience, Storytelling.

\section{INTRODUCTION}

A large part of our social life consists of sharing daily stories with other people. Storytelling is a key element of social interaction as it helps people to express their feelings and to establish a bond with others. However, sharing stories is extremely difficult for people with limited verbal ability, such as people suffering form (expressive) Aphasia. Consequently, Aphasia often leads to increased social isolation and

(C) The Author 2008.

Published by the British Computer Society depression. The premise of our project is that enabling Aphasics to share their daily stories will help them to become more active socially and to reengage in their preferred life style.

Aphasia is an acquired communication disorder that is caused by brain injury or trauma. Aphasia affects language comprehension and generation [6], so that people's ability to verbally express themselves suffers. Approximately 1 million people in the USA suffer from Aphasia. In an ageing population, an increasing percentage of people suffer from strokes. Approximately $30 \%$ of them will be diagnosized as being aphasic. Aphasia is a chronic condition that cannot be cured, although it can improve to some extent through therapy. People with Aphasia hence need to learn how to cope with their disability. Solutions that can accomplish a more effective communication in people with aphasia will not only empower these patients themselves, but can also help to reduce the burden on partners and caregivers.

Next to low-tech aids, such as drawing books, an increasing number of high-tech devices for assisting people with Aphasia in their daily communication have been proposed. Augmentative and Alternative Communication (AAC) Devices such as TouchSpeak [12] are widely used in Aphasia therapy and in a post-therapy period. Although there are different commercial devices today, they are not extremely successful due to several limitations. Since AAC devices only support need-based interaction, they are not sufficient as a tool to share daily stories. These devices often need to configured or set by the caregivers and/or by partners. Most AAC devices also tend to stigmatize their users, which implies that many aphasic persons prefer no to use them in social gatherings, i.e., outside their immediate environment of close relatives.

Recent research on Aphasia includes the ESI planner [11], an enhanced sound and image planner that help Aphasics to manage their schedule independently. Another recent system is a desktop-PDA [3] that supports communication through sound and images collected inside or outside of the home. The visual recipe book [13] is a specific application that assists people with Aphasia in cooking. These applications demonstrate that there is growing interest in the design research community to create systems that can assist Aphasics in their needs for daily communication.

Photography, as implemented in 'Aphasia Talks' [10] has been used to facilitate self-expression in Aphasics for the purpose of reintegration, socialization and recreation. However, user studies revealed that the collection of photos with a traditional camera was cumbersome for Aphasics, partly because many Aphasics also suffer from right-sided paralysis and/or have problems reading textual labels. 
Digital photographs have often been used for telling stories. One such application is iTell [9] which allows users to build stories by brainstorming, organizing, writing and adding personal media. This application is however not tailored to people with limited verbal ability, such as Aphasics. Another relevant application is a multimedia communication service that was developed for non-speaking people [7]. However, since this project focused on telling a story, it remained vague how the elements needed for a story should be collected. Therefore, there is a clear gap in research outlining how people with special needs can be enabled to collect the materials needed for story building. In our research we therefore primarily focused on how people with expressive Aphasia can be assisted while gathering story materials. Several studies have been published on the topic of capturing life experiences by both able and disabled people, such as the wearable camera SenseCam [5], online media weblog [8], group story sharing [2], etc. All applications have their own limitations in terms of collecting, processing, and building, retrieving and expressing experiences by a specific target group from the available materials.

This project is part of a research project that aims to investigate whether or not technology can help to reduce the social isolation of Aphasics, in particular those who have been discharged from the rehabilitation center and that need to continue their life with a serious disability. In our preliminary investigation, we focus on how Aphasics staying at home can relay stories about their daily experiences with others, such as their partner who is absent during the day. One argument for this choice is that ageing people often like to stay at home, as they consider home to be a sanctuary [4]. As many Aphasics also cannot pick up their earlier jobs, they are likely to spend more time at home than before. Developing their communication skills, and in doing so regaining confidence and a social position, is therefore likely to start at home. In our chosen context, we refer to storytelling as the reporting of daily activity and phrase our research goals as

- collecting the requirements for an in-home storytelling support system for Aphasics;

- gathering information on how different materials needed for story creation should preferably be collected;

- exploring alternatives for building and expressing stories from the gathered materials.

There are hence several distinct aspects that need to be addressed when designing a storytelling system, i.e., (a) (re)collecting materials, (b) building the story from these materials and (c) expressing the story to others or letting others explore the story by themselves. Each of these components creates a challenge of its own. In the current paper, we focus on passive capturing of story materials, that is, on how the home environment can help Aphasics by automatically capturing story materials for them. Since many Aphasics suffer up to a certain degree from right-handed paralysis, we hypothesize that passive capture can reduce the effortful interaction that might otherwise be required for collecting story materials. Of course, there is also a possible downside to passive collecting, i.e., that more interaction is required in later stages to sort and select collected materials.

\section{FIELD ENQUIRY}

Due to the nature of the target group, it is difficult to interview Aphasics or even engage them in a design process. Therefore, we have adopted a different methodology, similar to the one mentioned in [1]. We interviewed the proxies of the Aphasics, such as the caregivers (e.g., the speech therapist) and partners.
As a first step to knowing more about Aphasia we visited a local rehabilitation center and interviewed one of the speech therapists. The main discussion points were Aphasia and the capability of Aphasics, the social life of Aphasics, memory limitations in Aphasics, and the use of technology with Aphasics.

Slower information processing. Since the semantic system of Aphasics is damaged to some degree they have difficulty understanding especially abstract concepts. The semantic system however often recovers partly in the first couple of months after a stroke. Aphasics cannot process information as fast as most people and more often make mistakes. Processing simultaneous information is also harder for them. Aphasics often have problems concentrating for a very long time on one thing and they fatigue more easily. They can also have problems in taking initiatives.

Memory and Aphasia. The memory of people with Aphasia can also be affected, in particular the short term memory. New information is harder for them to retain. For instance, when Aphasics express themselves they can easily forget the original context and get lost. Consequently they lack a clue as to why they are expressing themselves, which in turn leads to a loss of coherence. Usually, their long-term memory is much less affected.

Recognize and recall. The speech therapists mentioned that Aphasics can understand photographs. Usually they don't have any problem in recognizing objects or people from photographs. However, it might be difficult for them if the image is (a) very detailed, (b) very small, (c) can refer to different contexts, i.e., has several different associations. Words are most abstract and hence more difficult to perceive than pictograms, which makes the latter easier for them to understand. Words can also be unambiguous. For instance, star could be a pop star or something in the sky. For Aphasics it might be hard to differentiate. Therefore, Asphasics often seek confirmation from the communication partner that they have done of interpreted something the right way.

Use of technology. Most of the time people having Aphasia can manage to do what they have to do. However, for some a schedule is needed. Many Aphasics at the local rehabilitation center that we visited use TouchSpeak [13] with a handheld device. Sometimes they put the device on their lap and use it with their left hand. While they are walking they have to carry the device. Speech therapists mentioned that TouchSpeak is suitable for those patients who can handle association between objects (groups of similar objects), Many Aphasics can learn to operate TouchSpeak, provided they get assistance (from the speech therapist) in personalization the device. Therapists help them to accomplish certain steps, which the Aphasics afterwards rehearse. However, the device has obvious limitations, such as a limited number of, practically oriented, sentences in which only a few words can be modified.

Aphasia and emotion. Usually Aphasics express their feelings through mimicry, sounds, yes or no, sign language and body language (not much). Aphasics spend around 9 months in the rehabilitation center. It is very difficult to create technology for Aphasia people, as the target group is very diverse. People in the Netherlands use much less gesturing than people in more southern countries, which implies that they are not used to communicating through gestures. Explaining how they feel is tough for them.

Partner dependency. Aphasics tend to look at their partners when they need to express something. Both the aphasic person 
and his partner are afraid of making mistakes. Aphasics often have problems follow a conversation at a normal pace, and need more time to catch up with what's going on. Partners in a conversation hence need to allow for time for the aphasic person to catch up.

\section{CONCEPT DESIGN}

Based on the findings from the first interview we have generated several concepts. These concepts emphasize the following features: (a) image-based communication, (b) independent expression of experience, (c) limited effort for collecting and sharing experience, (d) means for expressing emotions, (e) use of computing technology, and (f) involving the partner in creating and expressing a story. We have chosen three concepts for further exploration. The main goal was to express how Aphasics can report their daily activities and can share their daily experiences with their partner and friends. Each of the concepts is divided into two parts: (a) collection of the story materials, and (b) building and expressing the story.

Passive capturing through ubiquitous camera. In this concept, the house of the aphasic person is equipped with several wireless cameras that can capture images of ongoing activities. The ubiquitous cameras are integrated in the environment and capture all activities within their field of view. Low-quality images are filtered out, while the remaining images are stored in a computing device to make them available for later retrieval. The user doesn't need to do any explicit interaction with the camera since the intelligent camera uses pre-programmed algorithms. The partner (husband/wife/friend), together with the aphasic person, uses the stored images to reconstruct the activities of the day. They can both select relevant pictures and annotate them through touch/pen-based interaction. The Aphasic can express emotion through the emotion expressing panel of the supporting device (e.g. PDA, palmtop). The story can be saved for later retrieval.
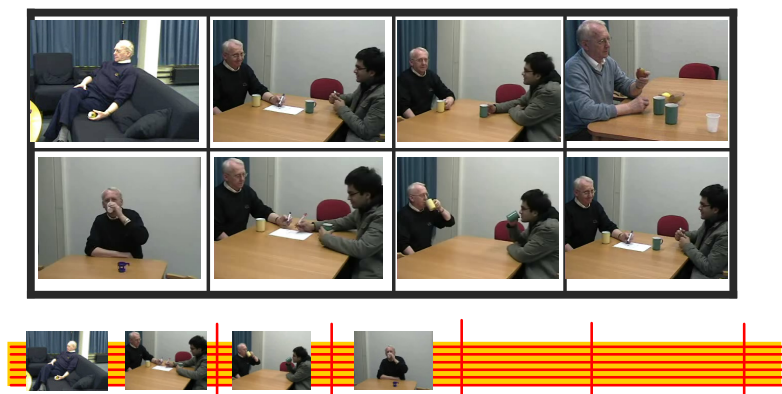

Figure 1. A sample storyboard for collecting (top) and building (bottom) a story.

Context dependent capturing of daily activities. In this concept, the capturing device installed at home takes photographs by active interaction in the environment. For instance, if the Aphasic person sits on the couch the system detects this activity and captures it. The captured photographs are tagged and saved according to the place and time. Similarly, the intelligent system can detect other activities that the aphasic carries out like taking medication or drinking coffee by means of sensors embodied in the objects being used. The stored images can be used to create a tangible story book by the Aphasics and their partners. They have a physical book like device where they add the captured images as part of their daily story.

Remind me. In this concept, the house is equipped with an inhome reminder system; which means that the system can prompt user actions. The system reminds the aphasic person of the planned activities that need to be done via an audio and/or visual reminder (a display device mounted on the wall). As soon as the aphasic person performs a particular activity the system captures that activity and saves it. The captured materials i.e. the photos are stored in the system. The reminder system provides a template, such as a calendar, for arranging photos, consequently helping in building the story.

\section{CONCEPT VALIDATION}

A concept booklet was prepared and was shown to two expert speech therapists and a social worker in two locations (one in an Aphasia rehabilitation center, the other one is a local Aphasia union). The concepts were shown as storyboards (for example see Figure 1) and through video prototypes. The storyboards were drawn and printed on paper. The video prototype was like role playing and showed an Aphasic person named John going through his daily activities such as taking medication, having coffee with his neighbor, watching TV, etc. The storytelling support system in his home collected evidence and the Aphasics reconstructed his day together with his wife Sara.

Overall the speech therapists were positive about the concepts. They appreciated the idea of sharing experience through storytelling. One constructive point was that this kind of solution would be helpful to Aphasics in a nursing home since they have very few things to do there. They advised an option for recording especially what other people say to the aphasic person (e.g. fragment of videos), so that this can be used as an element in the story later on. For instance, when someone visits them or even when their doctors say something to them, Aphasics might want it to be shown later to their partners. The system should have more functionality for the partner to elaborate and engage more intimately while building the story.

Concerning the first concept, there should be some way for the aphasic person to also control the system, for instance to stop recording at specific times. Use of a computer for expressing a story is fine as either Aphasic have prior experience with using a computer or they can get used to it during the rehabilitation period. They can learn a simple user interface since they already use a computerized tool, such as TouchSpeak for therapy purpose. The downside is that if there are more images it might be a problem for the Aphasics to deal with, in terms of choosing the images that will be the building blocks of the story.

For the second concept, the opinion was that it would be the most appropriate if the system can generate selected images based on a decision making process. On the other hand more modality needs to be added in the system and sound recording can be added with images.

With regards to Concept 3, speech therapists liked the visual reminder but had the opinion that this can be an audio visual reminder since Aphasics are not deaf. They mentioned that there should be a way to capture unplanned tasks/unexpected events and that communication should be two ways: between Aphasics and other people. Sometimes Aphasics do not feel any need to communicate and they need to be motivated to use the system. If the damage is in frontal part of their brain they lose the ability to take initiatives.

During the concept evaluation session, the speech therapists suggested that it is always reliable to use sound. Sound and visual cues in combination are a preferred medium. Use of multiple channels such as visual, gestures etc. as modalities for Aphasics will be beneficial. Speech therapists also pointed out that the partner plays a vital role in the life of the Aphasics. 
Therefore, any system for the Aphasics should also have functionalities/roles for their partners.

\section{INITIAL PROTOTYPING}

In the first phase of implementation, we are focusing on the first concept that is passive capture of story materials through ubiquitous camera and co-creating the past with their partner. However, the other concepts are worthy of consideration at a later stage. While trying to develop our prototype of the envisioned system, it is clear that there are several challenges, such as collecting the story materials passively and presenting those materials in an environment that can embellish the story.

Passive Capturing and Sampling. Since passive capture usually generates a huge collection of images, filtering them to feed into the story creation application is a main challenge. In the concept evaluation phase it was also revealed that providing more images could be overwhelming for the Aphasics. Therefore, we ran a simple experiment to see the possibilities of filtering images and providing only content rich images for a story. A camera was setup in an office environment for 8 hours and later the images were reviewed with colleagues to find out interesting images. It was found that with a simple webcam application running a motion detection utility a lot of images were captured in which a real object was not recognized. The images were then shown to some colleagues. They mentioned that images with an actor or where the face is visible are the most interesting. This session gave us an idea about the sampling algorithm of the acquired images.

User Interaction and User Interface Design. To present the acquired images for story building we built paper prototypes. The story building application is itself made of images/icons. Representative icons were chosen to make the menu option for representing different functions. The application has the option to add or delete or preview stories and in order to decorate the story it has the option for tagging, adding emotions and recording or adding sounds to a story.

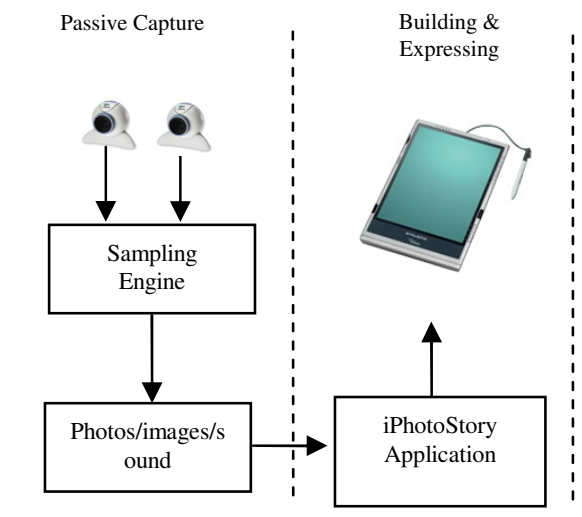

\section{CONCLUSION \& FUTURE WORK}

We have presented the initial exploration of the concepts and prototyping for designing a home-based storytelling system through passive capturing of story materials. The early exploration phase with speech therapists shows some interesting insights about storytelling for Aphasics especially what materials to collect and how much to collect and present to Aphasics for story creation. We will evaluate the paper prototyping with detailed interaction with the speech therapist and partner of the Aphasics. Afterwards the final prototyping will be implemented and will be evaluated with the potentials users and with their proxies.

\section{ACKNOWLEDGMENTS}

We are grateful to Reijndam Rehabilitation Center, Rotterdam; Aphasia Center, Rotterdam, and Rehabilitation Center Blixembosch, Eindhoven for their cooperation and guidance.

\section{REFERENCES}

[1] Allen, M., Leung, R., McGrenere, J., and Purves, B. 2008. Involving domain experts in assistive technology research, Universal Access in the Information Society, Springer Berlin / Heidelberg

[2] Appan, P., Sundaram, H., and Birchfield, D. 2004. Communicating everyday experiences. In Proceedings of the 1st ACM Workshop on Story Representation, Mechanism and Context, SRMC '04. ACM, New York, NY, 17-24.

[3] Boyd-Graber, J. L., Nikolova, S. S., Moffatt, K. A., Kin, K. C., Lee, J. Y., Mackey, L. W., Tremaine, M. M., and Klawe, M. M. 2006. Participatory design with proxies: developing a desktop-PDA system to support people with aphasia. In CHI '06. ACM, New York, NY, 151-160

[4] Cook, D. J. and Das, S. K. 2007. How smart are our environments? An updated look at the state of the art. Pervasive Mob. Comput. 3, 2 (Mar. 2007), 53-73.

[5] Gemmell, J., Williams, L., Wood, K., Lueder, R., and Bell, G. 2004. Passive capture and ensuing issues for a personal lifetime store. In Proceedings of the 1st ACM Workshop on Continuous Archival and Retrieval of Personal Experiences, CARPE'04. ACM, New York, NY, 48-55.

[6] Hillis, A. E. 2007. Aphasia: Progress in the last quarter of a century, Neurology, July 2007, 69:200-213

[7] Hine, Nick., Arnot, John L.,(2002). Assistive social interaction for non speaking people living in the community. Assets 2002, pp. 162-169

[8] Kelliher, A. 2004. Everyday cinema. In Proceedings of the 1st ACM Workshop on Story Representation, Mechanism and Context, SRMC '04. ACM, New York, NY, 59-62.

[9] Landry, B. M. and Guzdial, M. 2006. iTell: supporting retrospective storytelling with digital photos. In Proceedings of the 6th Conference on Designing interactive Systems, DIS '06. ACM, New York, NY, 160168.

[10] Levin T, Scott BM, Borders B, Hart K, Lee J, Decanini A. 2007. Aphasia Talks: photography as a means of communication, self-expression, and empowerment in persons with aphasia. Topics in stroke rehabilitation, 2007 Jan-Feb; 14(1):72-84.

[11] Moffatt, K., McGrenere, J., Purves, B., and Klawe, M. 2004. The participatory design of a sound and image enhanced daily planner for people with aphasia. In CHI '04. ACM, New York, NY, 407-414.

[12] Van de Sandt-Koenderman WM, Wiegers J, Wielaert SM, Duivenvoorden HJ, Ribbers GM. (2007). A computerised communication aid in severe aphasia: an exploratory study. Disability and Rehabilitation. 2007 Nov 30; 29(22):1701-9.

[13] Tee, K., Moffatt, K., Findlater, L., MacGregor, E., McGrenere, J., Purves, B., and Fels, S. S. 2005. A visual recipe book for persons with language impairments. In CHI '05. ACM, New York, NY, 501-510. 